\title{
On w-hyponormal operators
}

\author{
by \\ EungIL Ko (Seoul)
}

\begin{abstract}
We study some properties of w-hyponormal operators. In particular we show that some w-hyponormal operators are subscalar. Also we state some theorems on invariant subspaces of w-hyponormal operators.
\end{abstract}

1. Introduction. Let $\mathbf{H}$ be a complex Hilbert space, and denote by $\mathcal{L}(\mathbf{H})$ the algebra of all bounded linear operators on $\mathbf{H}$. If $T \in \mathcal{L}(\mathbf{H})$, we write $\sigma(T), \sigma_{\text {ap }}(T)$, and $\sigma_{\mathrm{p}}(T)$ for the spectrum, approximate point spectrum, and point spectrum of $T$, respectively.

An operator $T \in \mathcal{L}(\mathbf{H})$ is said to be $p$-hyponormal, $0<p \leq 1$, if $\left(T^{*} T\right)^{p} \geq$ $\left(T T^{*}\right)^{p}$ where $T^{*}$ is the adjoint of $T$. If $p=1, T$ is called hyponormal, and if $p=1 / 2, T$ is called semi-hyponormal. Semi-hyponormal operators were introduced by Xia (see $[\mathrm{Xi}]$ ), and $p$-hyponormal operators for a general $p$, $0<p<1$, have been studied by Aluthge. Any $p$-hyponormal operator is $q$ hyponormal if $q \leq p$ by Löwner's theorem (see [Lo]). But there are examples to show that the converse of the above statement is not true (see [Al]).

An arbitrary operator $T \in \mathcal{L}(\mathbf{H})$ has a unique polar decomposition $T=U|T|$, where $|T|=\left(T^{*} T\right)^{1 / 2}$ and $U$ is the appropriate partial isometry satisfying $\operatorname{ker} U=\operatorname{ker}|T|=\operatorname{ker} T$ and $\operatorname{ker} U^{*}=\operatorname{ker} T^{*}$. Associated with $T$ is a related operator $|T|^{1 / 2} U|T|^{1 / 2}$, called the Aluthge transform of $T$, and denoted by $\widetilde{T}$ throughout this paper.

An operator $T=U|T|$ (polar decomposition) in $\mathcal{L}(\mathbf{H})$ is w-hyponormal if $|\widetilde{T}| \geq|T| \geq\left|\widetilde{T}^{*}\right|$ where $|\widetilde{T}|=\left(\widetilde{T}^{*} \widetilde{T}\right)^{1 / 2}$. This class of operators was introduced by Aluthge and Wang (see [AW 1] and [AW 2]).

An operator $T \in \mathcal{L}(\mathbf{H})$ is said to satisfy the single-valued extension property if for any open subset $U$ in $\mathbb{C}$, the function

$$
z-T: \mathcal{O}(U, \mathbf{H}) \rightarrow \mathcal{O}(U, \mathbf{H})
$$

2000 Mathematics Subject Classification: 47B20, 47A11.

The author was supported by a Korea Research Foundation grant (KRF-2001-015DP0023). 
defined by the obvious pointwise multiplication is one-to-one, where $\mathcal{O}(U, \mathbf{H})$ denotes the Fréchet space of $\mathbf{H}$-valued analytic functions on $U$ with respect to uniform topology. If $T$ has the single-valued extension property, then for any $x \in \mathbf{H}$ there exists a unique maximal open set $\varrho_{T}(x)(\supset \varrho(T)$, the resolvent set) and a unique $\mathbf{H}$-valued analytic function $f$ defined in $\varrho_{T}(x)$ such that

$$
(T-\lambda) f(\lambda)=x, \quad \lambda \in \varrho_{T}(x) .
$$

An operator $T \in \mathcal{L}(\mathbf{H})$ is said to have the property $(\beta)$ if for every open subset $G$ of $\mathbb{C}$ and every sequence $f_{n}: G \rightarrow \mathbf{H}$ of $\mathbf{H}$-valued analytic functions such that $(T-\lambda) f_{n}(\lambda)$ converges uniformly to 0 in norm on compact subsets of $G, f_{n}(\lambda)$ converges uniformly to 0 in norm on compact subsets of $G$.

A bounded linear operator $S$ on $\mathbf{H}$ is called scalar of order $m$ if it has a spectral distribution of order $m$, i.e., if there is a continuous unital morphism of topological algebras

$$
\Phi: C_{0}^{m}(\mathbb{C}) \rightarrow \mathcal{L}(\mathbf{H})
$$

such that $\Phi(z)=S$, where as usual $z$ stands for the identity function on $\mathbb{C}$ and $C_{0}^{m}(\mathbb{C})$ stands for the space of compactly supported functions on $\mathbb{C}$, continuously differentiable of order $m, 0 \leq m \leq \infty$. An operator is subscalar if it is similar to the restriction of a scalar operator.

In this paper we study some properties of w-hyponormal operators. In particular we show that some w-hyponormal operators are subscalar. Also we study invariant subspaces of w-hyponormal operators.

2. Preliminaries. Let $d \mu(z)$ denote the planar Lebesgue measure. Fix a complex (separable) Hilbert space $\mathbf{H}$ and a bounded open disk $D$ of $\mathbb{C}$. We shall denote by $L^{2}(D, \mathbf{H})$ the Hilbert space of measurable functions $f: D \rightarrow \mathbf{H}$ such that

$$
\|f\|_{2, D}=\left\{\int_{D}\|f(z)\|^{2} d \mu(z)\right\}^{1 / 2}<\infty .
$$

The space of functions $f \in L^{2}(D, \mathbf{H})$ which are analytic on $D$ (i.e. $\bar{\partial} f=0$ ) is denoted by

$$
A^{2}(D, \mathbf{H})=L^{2}(D, \mathbf{H}) \cap \mathcal{O}(D, \mathbf{H}) .
$$

$A^{2}(D, \mathbf{H})$ is called the Bergman space for $D$. Note that $A^{2}(D, \mathbf{H})$ is complete (i.e. $A^{2}(D, \mathbf{H})$ is a Hilbert space). We denote by $P$ the orthogonal projection of $L^{2}(D, \mathbf{H})$ onto $A^{2}(D, \mathbf{H})$.

Let us now define a Sobolev type space called $W^{2}(D, \mathbf{H})$ where $D$ is a bounded disk in $\mathbb{C}$. $W^{2}(D, \mathbf{H})$ will be the space of those functions $f \in$ $L^{2}(D, \mathbf{H})$ whose derivatives $\bar{\partial} f, \bar{\partial}^{2} f$ in the sense of distributions still belong 
to $L^{2}(D, \mathbf{H})$. Endowed with the norm

$$
\|f\|_{W^{2}}^{2}=\sum_{i=0}^{2}\left\|\bar{\partial}^{i} f\right\|_{2, D}^{2},
$$

$W^{2}(D, \mathbf{H})$ becomes a Hilbert space contained continuously in $L^{2}(D, \mathbf{H})$.

Now for $f \in C_{0}^{2}(\mathbb{C})$, let $M_{f}$ denote the operator on $W^{2}(D, \mathbf{H})$ given by multiplication by $f$. It has a spectral distribution of order 2 , defined by the functional calculus

$$
\Phi_{M}: C_{0}^{2}(\mathbb{C}) \rightarrow \mathcal{L}\left(W^{2}(D, \mathbf{H})\right), \quad \Phi_{M}(f)=M_{f}
$$

Therefore, $M_{z}$ is a scalar operator of order 2 .

3. Single-valued extension property. In this section, we show that some w-hyponormal operators have the single-valued extension property. We also give an analogue of the single-valued extension property for $W^{2}(D, \mathbf{H})$ and some w-hyponormal operators $T$.

Recall that an operator $T \in \mathcal{L}(\mathbf{H})$ has finite ascent if for all $\lambda \in \mathbb{C}$ there is an $n \in \mathbb{N}$ such that $\operatorname{ker}(T-\lambda)^{n}=\operatorname{ker}(T-\lambda)^{n+1}$.

LEMMA 3.1. An operator $|T|^{1 / 2}$ is one-to-one if and only if the operator $|\widetilde{T}|^{1 / 2}$ is one-to-one.

Proof. Assume that $|T|^{1 / 2}$ is one-to-one. If $x \in \operatorname{ker}|\widetilde{T}|^{1 / 2}$, then $\widetilde{T} x=0$. Since $T\left(U|T|^{1 / 2}\right)=\left(U|T|^{1 / 2}\right) \widetilde{T}$, we have $|T|\left(U|T|^{1 / 2} x\right)=0$. Since $|T|^{1 / 2}$ is one-to-one, $x=0$.

Conversely, assume that $|\widetilde{T}|^{1 / 2}$ is one-to-one. If $x \in \operatorname{ker}|T|^{1 / 2}$, then $\widetilde{U}|\widetilde{T}| x=\widetilde{T} x=|T|^{1 / 2} U|T|^{1 / 2} x=0$. Since $|\widetilde{T}|^{1 / 2}$ is one-to-one, $x=0$.

THEOREM 3.2. If $T=U|T|$ (polar decomposition) is w-hyponormal with $0 \notin \sigma_{\mathrm{p}}\left(|T|^{1 / 2}\right)$, then $T$ has finite ascent.

Proof. Assume that $T$ is w-hyponormal with $0 \notin \sigma_{\mathrm{p}}\left(|T|^{1 / 2}\right)$. Then $\widetilde{T}$ is hyponormal from the definition of a w-hyponormal operator and [Al]. Since $\widetilde{\widetilde{T}}$ is hyponormal, $\operatorname{ker}(\widetilde{\widetilde{T}}-\lambda)=\operatorname{ker}(\widetilde{\widetilde{T}}-\lambda)^{2}$ for all $\lambda \in \mathbb{C}$. So it suffices to show that $\operatorname{ker}(\widetilde{T}-\lambda) \supset \operatorname{ker}(\widetilde{T}-\lambda)^{2}$. Let $\widetilde{T}=\widetilde{U}|\widetilde{T}|$ be the polar decomposition of $\widetilde{T}$ and let $x \in \operatorname{ker}(\widetilde{T}-\lambda)^{2}$. Since

$$
(\widetilde{T}-\lambda)^{2}|\widetilde{T}|^{1 / 2} x=|\widetilde{T}|^{1 / 2}(\widetilde{T}-\lambda)^{2} x=0,
$$

it follows from the hypothesis that

$$
|\widetilde{T}|^{1 / 2} x \in \operatorname{ker}(\widetilde{T}-\lambda)^{2}=\operatorname{ker}(\widetilde{T}-\lambda) .
$$

Hence

$$
|\widetilde{T}|^{1 / 2}(\widetilde{T}-\lambda) x=(\widetilde{T}-\lambda)|\widetilde{T}|^{1 / 2} x=0
$$


Since $|\widetilde{T}|^{1 / 2}$ is one-to-one by Lemma 3.1, $(\widetilde{T}-\lambda) x=0$. Hence $x \in \operatorname{ker}(\widetilde{T}-\lambda)$. Thus $\widetilde{T}$ has finite ascent. By a similar method we deduce that $T$ has finite ascent.

COROllary 3.3. If $T=U|T|$ (polar decomposition) is a w-hyponormal operator with $0 \notin \sigma_{\mathrm{p}}\left(|T|^{1 / 2}\right)$, then $T$ has the single-valued extension property.

Proof. This follows from Theorem 3.2 and [La].

Corollary 3.4. Let $T=U|T|$ (polar decomposition) be a w-hyponormal operator with $0 \notin \sigma_{\mathrm{p}}\left(|T|^{1 / 2}\right)$. If $f: G \rightarrow \mathbb{C}$ is an analytic function nonconstant on every component of $G$ where $G$ is open and $G \supset \sigma(T)$, then $f(T)$ has the single-valued extension property.

Proof. Since $T$ has the single-valued extension property by Corollary 3.3, the assertion follows from [CF, Theorem 1.1.5].

Recall that an $X \in \mathcal{L}(\mathbf{H}, \mathbf{K})$ is called a quasi-affinity if it has trivial kernel and dense range. An operator $A \in \mathcal{L}(\mathbf{H})$ is said to be a quasi-affine transform of an operator $T \in \mathcal{L}(\mathbf{K})$ if there is a quasi-affinity $X \in \mathcal{L}(\mathbf{H}, \mathbf{K})$ such that $X A=T X$.

COROllary 3.5. Let $T=U|T|$ (polar decomposition) be a w-hyponormal operator with $0 \notin \sigma_{\mathrm{p}}\left(|T|^{1 / 2}\right)$. If $A$ is any quasi-affine transform of $T$, then $A$ has the single-valued extension property.

Proof. From [La], it suffices to show that $\operatorname{ker}(A-\lambda)^{2} \subset \operatorname{ker}(A-\lambda)$ for all $\lambda \in \mathbb{C}$. Let $X$ be a quasi-affinity such that $X A=T X$. If $x \in \operatorname{ker}(A-\lambda)^{2}$, then $X(A-\lambda)^{2} x=0$. Hence $(T-\lambda)^{2} X x=0$. Since $\operatorname{ker}(T-\lambda)^{2}=\operatorname{ker}(T-\lambda)$ from Theorem 3.2, $(T-\lambda) X x=0$. Hence $X(A-\lambda) x=0$. Since $X$ is one-to-one, $x \in \operatorname{ker}(A-\lambda)$.

The next result gives an analogue of the single-valued extension property for $W^{2}(D, \mathbf{H})$ and some w-hyponormal operators $T$.

ThEOREM 3.6. Let $T=U|T|$ (polar decomposition) be a w-hyponormal operator with $0 \notin \sigma_{\mathrm{p}}\left(|T|^{1 / 2}\right)$ in $\mathcal{L}(\mathbf{H})$ and let $D$ be an arbitrary bounded disk in $\mathbb{C}$. Then the operator

$$
T-z: W^{2}(D, \mathbf{H}) \rightarrow W^{2}(D, \mathbf{H})
$$

is one-to-one.

Proof. Let $f \in W^{2}(D, \mathbf{H})$ be such that $(T-z) f=0$. Then

$$
(\widetilde{T}-z)|T|^{1 / 2} f=0 .
$$

Let $\widetilde{T}=\widetilde{U}|\widetilde{T}|$ be the polar decomposition of $\widetilde{T}$. Then from (1) we get

$$
(\widetilde{T}-z)|\widetilde{T}|^{1 / 2}|T|^{1 / 2} f=0 .
$$


Since $\widetilde{T}$ is hyponormal from the definition of a w-hyponormal operator and [Al], [Pu, Corollary 2.2] implies that

$$
|\widetilde{T}|^{1 / 2}|T|^{1 / 2} f=P\left(|\widetilde{T}|^{1 / 2}|T|^{1 / 2} f\right)
$$

where $P$ is the orthogonal projection of $L^{2}(D, \mathbf{H})$ onto $A^{2}(D, \mathbf{H})$. From (2) and (3), we have

$$
(\widetilde{T}-z) P\left(|\widetilde{T}|^{1 / 2}|T|^{1 / 2} f\right)=0 .
$$

Since $\widetilde{T}$ has the single-valued extension property,

$$
|\widetilde{T}|^{1 / 2}|T|^{1 / 2} f=P\left(|\widetilde{T}|^{1 / 2}|T|^{1 / 2} f\right)=0 .
$$

Since $|T|^{1 / 2}$ is one-to-one, $|\widetilde{T}|^{1 / 2}$ is also one-to-one from Lemma 3.1. Hence $f=0$.

Corollary 3.7. Let $T=U|T|$ (polar decomposition) be any w-hyponormal operator in $\mathcal{L}(\mathbf{H})$. If $T$ has no nontrivial invariant subspace, then the operator

$$
T-z: W^{2}(D, \mathbf{H}) \rightarrow W^{2}(D, \mathbf{H})
$$

is one-to-one.

Proof. Since $T$ has no nontrivial invariant subspace for $T, \operatorname{ker} T=\{0\}$. Hence ker $|T|^{1 / 2}=\{0\}$. By Theorem 3.6, $T-z$ is one-to-one.

Corollary 3.8. Let $T_{1}$ and $T_{3}$ be w-hyponormal operators with $0 \notin$ $\sigma_{\mathrm{p}}\left(\left|T_{1}\right|^{1 / 2}\right) \cup \sigma_{\mathrm{p}}\left(\left|T_{3}\right|^{1 / 2}\right)$. Then

$$
\begin{aligned}
A-z=\left(\begin{array}{cc}
T_{1}-z & T_{2} \\
0 & T_{3}-z
\end{array}\right): & \\
& W^{2}(D, \mathbf{H}) \oplus W^{2}(D, \mathbf{H}) \rightarrow W^{2}(D, \mathbf{H}) \oplus W^{2}(D, \mathbf{H})
\end{aligned}
$$

is one-to-one.

Proof. Let $f=f_{1} \oplus f_{2} \in W^{2}(D, \mathbf{H}) \oplus W^{2}(D, \mathbf{H})$ be such that $(A-z) f=0$. Then

$$
\left(\begin{array}{cc}
T_{1}-z & T_{2} \\
0 & T_{3}-z
\end{array}\right)\left(\begin{array}{l}
f_{1} \\
f_{2}
\end{array}\right)=\left(\begin{array}{c}
\left(T_{1}-z\right) f_{1}+T_{2} f_{2} \\
\left(T_{3}-z\right) f_{2}
\end{array}\right)=\left(\begin{array}{l}
0 \\
0
\end{array}\right)
$$

So we have

$$
\begin{aligned}
\left(T_{1}-z\right) f_{1}+T_{2} f_{2} & =0 \\
\left(T_{3}-z\right) f_{2} & =0 .
\end{aligned}
$$

By Theorem 3.6 and (5), $f_{2}=0$. Hence from (4) we have $\left(T_{1}-z\right) f_{1}=0$. Again by Theorem 3.6, $f_{1}=0$. Thus $f=0$.

4. Subscalarity. In this section we show that some w-hyponormal operators have scalar extensions. 
Lemma 4.1. An operator $|T|^{1 / 2}$ is bounded below if and only if the operator $|\widetilde{T}|^{1 / 2}$ is bounded below.

Proof. If $|T|^{1 / 2}$ is bounded below, then there exists $c>0$ such that $\left\||T|^{1 / 2} x\right\| \geq c\|x\|$ for all $x \in \mathbf{H}$. An easy calculation shows that $\|\widetilde{T} x\|=$ $\left\||T|^{1 / 2} U|T|^{1 / 2} x\right\| \geq c^{2}\|x\|$ for all $x \in \mathbf{H}$. Hence $\|\widetilde{T} \mid x\| \geq c^{2}\|x\|$ for all $x \in \mathbf{H}$. Thus $|\widetilde{T}|^{1 / 2}$ is bounded below.

Conversely, if $|\widetilde{T}|^{1 / 2}$ is bounded below, then it is clear that $\widetilde{T}$ is bounded below. Since $\sigma_{\text {ap }}(T)=\sigma_{\text {ap }}(\widetilde{T})$ by [JKP], $T$ is bounded below. Hence $|T|$ is bounded below. So we conclude that $|T|^{1 / 2}$ is bounded below.

COROLLARY 4.2. An operator $|T|^{1 / 2}$ has closed range if and only if the operator $|\widetilde{T}|^{1 / 2}$ has closed range.

Proof. This is clear from Lemma 4.1.

Lemma 4.3. Let $T \in \mathcal{L}(\mathbf{H})$ be a semi-hyponormal operator. If $\left\{f_{n}\right\}$ is a sequence in $L^{2}(D, \mathbf{H})$ such that $\lim _{n \rightarrow \infty}\left\|(T-z) f_{n}\right\|_{2, D}=0$ for all $z \in D$, then $\lim _{n \rightarrow \infty}\left\|(T-z)^{*} f_{n}\right\|_{2, D}=0$.

Proof. Assume that $\left\{f_{n}\right\}$ is as in the hypothesis. Let $Q=|T|-\left|T^{*}\right|$, $z=\varrho e^{i \theta}, 0<\varrho$, and $\left|e^{i \theta}\right|=1$ where $\left|T^{*}\right|=\left(T T^{*}\right)^{1 / 2}$. Since $T$ is semihyponormal, [Xi, Lemma 2.1] implies

$$
\left\{\begin{array}{l}
\lim _{n \rightarrow \infty}\left\|(|T|-\varrho) f_{n}\right\|_{2, D}=0, \\
\lim _{n \rightarrow \infty} \varrho \|\left(|T|^{1 / 2}\left(U-e^{i \theta}\right)^{*} f_{n} \|_{2, D}=0,\right. \\
\lim _{n \rightarrow \infty} \varrho\left\langle Q f_{n}, f_{n}\right\rangle=0 .
\end{array}\right.
$$

Since

$$
(T-z)^{*} f_{n}=|T|^{1 / 2}\left[|T|^{1 / 2}\left(U-e^{i \theta}\right)^{*} f_{n}\right]+e^{-i \theta}\left[(|T|-\varrho) f_{n}\right],
$$

we have

$$
\left\|(T-z)^{*} f_{n}\right\|_{2, D} \leq\left\||T|^{1 / 2}\right\| \cdot \|\left(|T|^{1 / 2}\left(U-e^{i \theta}\right)^{*} f_{n}\left\|_{2, D}+\right\|(|T|-\varrho) f_{n} \|_{2, D} .\right.
$$

This completes the proof.

Lemma 4.4. Let $T=U|T|$ (polar decomposition) be a w-hyponormal operator with $0 \notin \sigma_{\mathrm{ap}}\left(|T|^{1 / 2}\right)$, and let $D$ be a bounded disk which contains $\sigma(T)$. Then the map $V: \mathbf{H} \rightarrow H(D)$ defined by

$$
V h=1 \widetilde{\otimes} h\left(\equiv 1 \otimes h+\overline{(T-z) W^{2}(D, \mathbf{H})}\right)
$$

is one-to-one and has closed range, where $1 \otimes h$ denotes the constant function sending any $z \in D$ to $h$ and $H(D):=W^{2}(D, \mathbf{H}) / \overline{(T-z) W^{2}(D, \mathbf{H})}$.

Proof. Let $h_{n} \in \mathbf{H}$ and $f_{n} \in W^{2}(D, \mathbf{H})$ be sequences such that

$$
\lim _{n \rightarrow \infty}\left\|(T-z) f_{n}+1 \otimes h_{n}\right\|_{W^{2}}=0 .
$$


Then by the definition of the norm of Sobolev space, (6) implies

$$
\lim _{n \rightarrow \infty}\left\|(U|T|-z) \bar{\partial}^{i} f_{n}\right\|_{2, D}=0
$$

for $i=1,2$. Since $\widetilde{T}=|T|^{1 / 2} U|T|^{1 / 2}$,

$$
\lim _{n \rightarrow \infty}\left\|(\widetilde{T}-z) \bar{\partial}^{i}\left(|T|^{1 / 2} f_{n}\right)\right\|_{2, D}=0
$$

for $i=1,2$. Let $\widetilde{T}=\widetilde{U}|\widetilde{T}|$ be the polar decomposition of $\widetilde{T}$. Then from (8) we have, for $i=1,2$,

$$
\lim _{n \rightarrow \infty}\left\|(\widetilde{\widetilde{T}}-z) \bar{\partial}^{i}\left(|\widetilde{T}|^{1 / 2}|T|^{1 / 2} f_{n}\right)\right\|_{2, D}=0 .
$$

Since $\widetilde{T}$ is hyponormal, by [Pu, Corollary 2.2],

$$
\lim _{n \rightarrow \infty}\left\|(I-P)\left(|\widetilde{T}|^{1 / 2}|T|^{1 / 2} f_{n}\right)\right\|_{2, D}=0
$$

where $P$ denotes the orthogonal projection of $L^{2}(D, \mathbf{H})$ onto $A^{2}(D, \mathbf{H})$. From (6) and (10) we get

$$
\lim _{n \rightarrow \infty}\left\|(\widetilde{T}-z) P\left(|\widetilde{T}|^{1 / 2}|T|^{1 / 2} f_{n}\right)+1 \otimes|\widetilde{T}|^{1 / 2}|T|^{1 / 2} h_{n}\right\|_{2, D}=0 .
$$

Let $\Gamma$ be a curve in $D$ surrounding $\sigma(T)(=\sigma(\widetilde{T})=\sigma(\widetilde{\widetilde{T}})$ by [JKP $])$. Then for $z \in \Gamma$,

$$
\lim _{n \rightarrow \infty}\left\|P\left(|\widetilde{T}|^{1 / 2}|T|^{1 / 2} f_{n}\right)(z)+(\widetilde{T}-z)^{-1}\left(1 \otimes|\widetilde{T}|^{1 / 2}|T|^{1 / 2} h_{n}\right)\right\|=0
$$

uniformly, from (11). Hence

$$
\lim _{n \rightarrow \infty}\left\|\frac{1}{2 \pi i} \int_{\Gamma} P\left(|\widetilde{T}|^{1 / 2}|T|^{1 / 2} f_{n}\right)(z) d z+|\widetilde{T}|^{1 / 2}|T|^{1 / 2} h_{n}\right\|=0 .
$$

But by Cauchy's theorem,

$$
\frac{1}{2 \pi i} \int_{\Gamma} P\left(|\widetilde{T}|^{1 / 2}|T|^{1 / 2} f_{n}\right)(z) d z=0 .
$$

Hence $\lim _{n \rightarrow \infty}|\widetilde{T}|^{1 / 2}|T|^{1 / 2} h_{n}=0$. Since $0 \notin \sigma_{\text {ap }}\left(|T|^{1 / 2}\right)$, by Lemma 4.1, $|\widetilde{T}|^{1 / 2}|T|^{1 / 2}$ is bounded below. Hence $\lim _{n \rightarrow \infty} h_{n}=0$. Thus the map $V$ is one-to-one and has closed range.

THEOREM 4.5. If $T=U|T|$ (polar decomposition) is a w-hyponormal operator with $0 \notin \sigma_{\text {ap }}\left(|T|^{1 / 2}\right)$, then $T$ is a subscalar operator of order 2 .

Proof. Suppose that $T=U|T|$ (polar decomposition) is a w-hyponormal operator with $0 \notin \sigma_{\text {ap }}\left(|T|^{1 / 2}\right)$. Consider an arbitrary bounded open disk $D$ in the complex plane $\mathbb{C}$ and the quotient space

$$
H(D)=W^{2}(D, \mathbf{H}) / \overline{(T-z) W^{2}(D, \mathbf{H})}
$$


endowed with the Hilbert space norm. The class of a vector $f$ or an operator $A$ on $H(D)$ will be denoted by $\widetilde{f}$, respectively $\widetilde{A}$. Let $M$ be the operator of multiplication by $z$ on $W^{2}(D, \mathbf{H})$. As noted at the end of Section $2, M$ is a scalar operator of order 2 and has a spectral distribution $\Phi$. Let $S \equiv \widetilde{M}$. Since $\overline{(T-z) W^{2}(D, \mathbf{H})}$ is invariant under every operator $M_{f}, f \in C^{2}(D)$, we infer that $S$ is a scalar operator of order 2 with spectral distribution $\widetilde{\Phi}$.

Consider the natural map $V: \mathbf{H} \rightarrow H(D)$ defined by $V h=(1 \otimes h)^{\sim}$ for $h \in \mathbf{H}$, where $1 \otimes h$ denotes the constant function identically equal to $h$. Note that $V T=S V$. In particular $\operatorname{ran} V$ is an invariant subspace for $S$. Since $V$ is one-to-one and has closed range by Lemma 4.4, $T$ is a subscalar operator of order 2.

COROLlaRY 4.6. Invertible w-hyponormal operators are subscalar of order 2 .

Proof. Let $T=U|T|$ (polar decomposition) be any invertible w-hyponormal operator. Then $|T|$ is invertible and $U$ is unitary. By [Ru, Thm. 12.33], $|T|^{1 / 2}$ is invertible. Since $|T|^{1 / 2}$ is positive, $\sigma\left(|T|^{1 / 2}\right)=\sigma_{\text {ap }}\left(|T|^{1 / 2}\right)$. Hence $0 \notin \sigma_{\text {ap }}\left(|T|^{1 / 2}\right)$. By Theorem 4.5, $T$ is a subscalar operator of order 2 .

COROllary 4.7. If $T=U|T|$ (polar decomposition) is a w-hyponormal operator with $0 \notin \sigma_{\mathrm{ap}}\left(|T|^{1 / 2}\right)$, then $T$ has Bishop's property $(\beta)$.

COROllary 4.8. Let $T=U|T|$ (polar decomposition) be a w-hyponormal operator with $0 \notin \sigma_{\mathrm{ap}}\left(|T|^{1 / 2}\right)$. If $A \in \mathcal{L}(\mathbf{H})$ is any quasi-affine transform of $T$, then $\sigma(T) \subseteq \sigma(A)$.

Proof. This follows from Corollary 4.7 and [Ko 1, Theorem 3.2].

Corollary 4.9. Let $T=U|T|$ (polar decomposition) be a w-hyponormal operator with $0 \notin \sigma_{\mathrm{ap}}\left(|T|^{1 / 2}\right)$ and let $f$ be a function analytic in a neighborhood of $\sigma(T)$. With the notation of the proof of Theorem 4.5, $\operatorname{Vf}(T)=$ $f(S) V$, where $f \mapsto f(T)$ is the functional calculus morphism.

Proof. This follows from a general property of the analytic functional calculus.

5. Theorems on invariant subspaces. In this section we study invariant subspaces of w-hyponormal operators. Recall that if $U$ is a nonempty open set in $\mathbb{C}$ and if $\Omega \subset U$ has the property that

$$
\sup _{\lambda \in \Omega}|f(\lambda)|=\sup _{\beta \in U}|f(\beta)|
$$

for every function $f$ in $H^{\infty}(U)$ (i.e. for all $f$ bounded and holomorphic on $U$ ), then $\Omega$ is said to be dominating for $U$.

The next theorem is a generalization of Scott Brown's theorem. 
THEOREM 5.1. Suppose that $T$ is an arbitrary w-hyponormal operator and there exists a nonempty open set $U$ in $\mathbb{C}$ such that $\sigma(T) \cap U$ is dominating for $U$. Then $T$ has a nontrivial invariant subspace.

Proof. If $T$ is not a quasi-affinity, then $0 \in \sigma_{\mathrm{p}}(T) \cup \sigma_{\mathrm{p}}\left(T^{*}\right)$. So it is trivial that $T$ has a nontrivial invariant subspace. Let $T$ be a quasi-affinity. Since $\widetilde{T}$ is semi-hyponormal from the definition of a w-hyponormal operator, [JKP, Theorem 1.24] implies that $\widetilde{T}$ has a nontrivial invariant subspace. By [JKP, Theorem 1.15], $T$ has a nontrivial invariant subspace.

The following theorem is a generalization of Berger's theorem.

TheOREM 5.2. Let $T$ be an arbitrary w-hyponormal operator. Then there exists a positive integer $K$ such that for all positive integers $k \geq K, T^{k}$ has a nontrivial invariant subspace.

Proof. If $T$ is not a quasi-affinity, then the result is trivial. Suppose that $T$ is a quasi-affinity. Since $\widetilde{T}$ is semi-hyponormal from the definition of a whyponormal operator, by [JKP, Theorem 1.25] there exists a positive integer $K$ such that for all positive integers $k \geq K,(\widetilde{T})^{k}$ has a nontrivial invariant subspace $\mathcal{M}_{k}$. Since $U|T|^{1 / 2}(\widetilde{T})^{j}=T^{j} U|T|^{1 / 2}$ and $\mathcal{M}_{k} \in \operatorname{Lat}\left((\widetilde{T})^{k}\right)$ for $k \geq K$,

$$
T^{k} U|T|^{1 / 2} \mathcal{M}_{k}=U|T|^{1 / 2}(\widetilde{T})^{k} \mathcal{M}_{k} \subset U|T|^{1 / 2} \mathcal{M}_{k}, \quad k \geq K .
$$

By [JKP, Theorem 1.15],

$$
\{0\} \neq\left(U|T|^{1 / 2} \mathcal{M}_{k}\right)^{-} \neq \mathbf{H} .
$$

Therefore, $\left(U|T|^{1 / 2} \mathcal{M}_{k}\right)^{-}$is the desired invariant subspace for $T^{k}$.

Recall that a closed subspace of $\mathbf{H}$ is said to be hyperinvariant for $T$ if it is invariant under every operator in the commutant $\{T\}^{\prime}$ of $T$.

THEOREM 5.3. Suppose that $T$ is an arbitrary w-hyponormal operator and

$$
\lim _{n \rightarrow \infty}\left\|T^{n} h\right\|^{1 / n}<\|T\|
$$

for some nonzero $h \in \mathbf{H}$. Then $T$ has a nontrivial hyperinvariant subspace.

Proof. If $T$ is an arbitrary w-hyponormal operator, then by [AW 2],

$$
\|T h\|^{2} \leq\left\|T^{2} h\right\| \cdot\|h\|
$$

for all $h \in \mathbf{H}$. Hence [Bo, Remark] implies that $T$ has a nontrivial hyperinvariant subspace.

Recall that an operator $T \in \mathcal{L}(\mathbf{H})$ is decomposable provided that, for each open cover $\{U, V\}$ of $\mathbb{C}$, there exist closed $T$-invariant subspaces $Y, Z$ of $\mathbf{H}$ such that $\mathbf{H}=Y+Z, \sigma\left(\left.T\right|_{Y}\right) \subset U$, and $\sigma\left(\left.T\right|_{Z}\right) \subset V$. Here, $\left.T\right|_{Y}$ denotes the restriction of $T$ to $Y$. 
LEMMA 5.4 ([LW, Lemma 3.6.1]). If $T$ is subscalar, then for all closed $F$ in $\mathbb{C}, H_{T}(F)$ is the linear span of all manifolds $Z$ in $\mathbf{H}$ satisfying $(\lambda-T) Z$ $=Z$ for all $\lambda \notin F$, where $H_{T}(F)=\{x \in \mathbf{H}: x=(\lambda-T) f(\lambda)$ for some analytic $f: \mathbb{C} \backslash F \rightarrow \mathbf{H}\}$.

THEOREM 5.5. Let $T$ be a w-hyponormal operator with $0 \notin \sigma_{\text {ap }}\left(|T|^{1 / 2}\right)$ and let $T \neq \lambda I$ for all $\lambda \in \mathbb{C}$. If $S$ is a decomposable quasi-affine transform of $T$, then $T$ has a nontrivial hyperinvariant subspace.

Proof. Assume that $X$ is a quasi-affinity such that $X S=T X$ where $S$ is decomposable. If $T$ has no nontrivial hyperinvariant subspace, we may assume that $\sigma_{\mathrm{p}}(T)=\emptyset$ and $H_{T}(F)=\{0\}$ for each closed $F$ proper in $\sigma(T)$ by Lemma 5.4. Let $\{U, V\}$ be an open cover of $\mathbb{C}$ with $\sigma(T) \backslash \bar{U} \neq \emptyset$ and $\sigma(T) \backslash \bar{V} \neq \emptyset$. Then

$$
X \mathbf{H}=X H_{S}(\bar{U})+X H_{S}(\bar{V}) \subseteq H_{T}(\bar{U})+H_{T}(\bar{V})=\{0\} .
$$

So we have a contradiction.

Acknowledgements. The author wishes to thank the referee for a careful reading of the original draft.

\section{References}

[Al] A. Aluthge, On p-hyponormal operators for $0<p<1$, Integral Equations Operator Theory 13 (1990), 307-315.

[AW 1] A. Aluthge and D. Wang, w-hyponormal operators, ibid. 36 (2000), 1-10.

[AW 2] -, 一, w-hyponormal operators II, ibid. 37 (2000), 324-331.

[Bo] P. Bourdon, Orbits of hyponormal operators, Michigan Math. J. 44 (1997), 345353.

[Br] S. W. Brown, Hyponormal operators with thick spectrum have invariant subspaces, Ann. of Math. 125 (1987), 93-103.

[CF] I. Colojoară and C. Foiaş, Theory of Generalized Spectral Operators, Gordon and Breach, New York, 1968.

[JKP] I. Jung, E. Ko and C. Pearcy, Aluthge transforms of operators, Integral Equations Operator Theory 38 (2000), 437-448.

[Ko 1] E. Ko, On a Clary theorem, Bull. Korean Math. Soc. 33 (1996), 29-33.

[Ko 2] -, On p-hyponormal operators, Proc. Amer. Math. Soc. 128 (2000), 775-780.

[LW] R. Lange and S. Wang, New Approaches in Spectral Decomposition, Contemp. Math. 128, Amer. Math. Soc., 1992.

[La] K. B. Laursen, Operators with finite ascent, Pacific J. Math. 152 (1992), 323-336.

[Lo] K. Löwner, Über monotone Matrixfunktionen, Math. Z. 38 (1934), 177-216.

[MP] M. Martin and M. Putinar, Lectures on Hyponormal Operators, Oper. Theory Adv. Appl. 39, Birkhäuser, Boston, 1989.

[Pu] M. Putinar, Hyponormal operators are subscalar, J. Operator Theory 12 (1984), 385-395.

[Ru] W. Rudin, Functional Analysis, McGraw-Hill, 1973. 
[Xi] D. Xia, Spectral Theory of Hyponormal Operators, Oper. Theory Adv. Appl. 10, Birkhäuser, Boston, 1983.

Department of Mathematics

Ewha Women's University

Seoul 120-750, South Korea

E-mail: eiko@mm.ewha.ac.kr

Received May 7, 2002

Revised version October 24, 2002 